\title{
Quantum Particles as 3D Differentiable Manifolds
}

\author{
Vu B Ho ${ }^{*}$ \\ Advanced Study, 9 Adela Court, Mulgrave, Victoria 3170, Australia \\ *Corresponding author: vubho@bigpond.net.au
}

Received August 07, 2018; Revised September 12, 2018; Accepted September 26, 2018

\begin{abstract}
As shown in our work on spacetime structures of quantum particles, Schrödinger wavefunctions in quantum mechanics can be utilised to construct the geometric structures of quantum particles which are considered to be three-dimensional differentiable manifolds. In this work we will extend this kind of geometric formulation of quantum particles by showing that wavefunctions that are normally used to describe wave phenomena in classical physics can in fact also be utilised to represent three-dimensional differentiable manifolds which in turns are identified with quantum particles. We show that such identification can be achieved by using a three-dimensional wave equation to construct three-dimensional differentiable manifolds that are embedded in a four-dimensional Euclidean space. In particular, the dual character that is resulted from the identification of a wavefunction with a three-dimensional differentiable manifold may provide a classical basis to interpret the wave-particle duality in quantum mechanics.
\end{abstract}

Keywords: quantum particle, wave equation, 3D differentiable manifold, general relativity, wave mechanics, wave-particle duality

Cite This Article: Vu B Ho, "Quantum Particles as 3D Differentiable Manifolds.” International Journal of Physics, vol. 6, no. 5 (2018): 139-146. doi: 10.12691/ijp-6-5-1.

\section{Introduction}

One of the most confusing concepts that we encounter in quantum mechanics is the notion of wave-particle duality. The idea emerges when we try to formulate the dynamics of a quantum particle using wave equations that are formulated in classical physics to describe classical wave phenomena [1]. Amazingly, beyond the expectation of the perception of physical existence in classical physics, the quantum formulation complies with the dual properties of quantum particles. Depending on physical settings, a quantum object exhibits both seemingly irreconcilable characters of a physical object defined in classical physics, that is the distinguished character that is associated with a particle and that with a wave. How can a single physical object manifest the wave character which can only be formed by a medium which is a collection of physical objects? According to Einstein's perception, physics expressed in terms of particles and their mutual interactions should be formulated according to the intrinsic geometric structures of spacetime, similar to the geometric formulation of his general relativity [2]. This has led to our speculation that quantum particles are extended physical objects which possess the mathematical structure of a three-dimensional differentiable manifold that are embedded in a four-dimensional Euclidean space [3]. Even with the identification of quantum particles with three-dimensional differentiable manifolds, there still remains the question of how to describe the wave character of these differentiable manifolds. A straightforward answer to the posed question is the differentiable manifolds must be solutions of a wave equation so that if quantum particles are identified with these differentiable manifolds then they can manifest wave characteristics. In this work we will endeavour to justify this answer by examining wave equations that are used to describe the wave motion of physical objects. As illustrations and visualisations, first we discuss the wave motion of a string in Section 2 in two-dimensional Euclidean space and a membrane in Section 3 in threedimensional Euclidean spaces and then, most importantly, in Section 4 we will discuss the wave motion of a vibrating solid ball described by a wave equation whose solutions are not considered to describe physical phenomena such as fluids and acoustics but differentiable manifolds embedded in a four-dimensional Euclidean space. In Sections 2 and 3 we show that the curvature of a curve and the Ricci scalar curvature of a surface can be determined by a wavefunction, but we don't have a relationship between the Ricci scalar curvature and a 3D wavefunction therefore as an illustration in Section 5 we recapture how Schrödinger wavefunctions can be used to determine the Ricci scalar curvature. Finally, in Section 6 we discuss geometric interactions in which decomposed cells from a CW complex could be associated with forces encountered in physical dynamics. In particular we show that all quantum particles can be formed from mass points joined by contact forces which are associated with the decomposition of 0 -cells from a CW complex which is identified with a quantum particle. 


\section{One-dimensional Wave Associated with a Vibrating String}

Consider the vibration of a string $D=\{0<x<L\}$ that satisfies the wave equation

$$
\frac{1}{c^{2}} \frac{\partial^{2} \psi}{\partial t^{2}}-\frac{\partial^{2} \psi}{\partial x^{2}}=0
$$

with boundary conditions $\psi(0, t)=0, \psi(L, t)=0$ and initial conditions $\psi(x, 0)=f(x), \partial \psi / \partial t(x, 0)=g(x)$. Then the general solution to the wave equation given in Equation (1) can be found as [4]

$$
\psi(x, t)=\sum_{n=1}^{\infty}\left(A_{n} \cos \alpha_{n} t+B_{n} \sin \alpha_{n} t\right) \sin \frac{n \pi x}{L}
$$

where

$$
\begin{gathered}
A_{n}=\frac{2}{L} \int_{o}^{L} f(x) \sin \frac{n \pi x}{L} d x \\
B_{n}=\frac{2}{c n \pi} \int_{o}^{L} g(x) \sin \frac{n \pi x}{L} d x
\end{gathered}
$$

By differentiating the wavefunction given in Equation (2), we obtain

$$
\begin{gathered}
\frac{d \psi}{d x}=\sum_{n=1}^{\infty}\left(\frac{n \pi}{L}\right)\left(A_{n} \cos \alpha_{n} t+B_{n} \sin \alpha_{n} t\right) \cos \frac{n \pi x}{L} \\
\frac{d^{2} \psi}{d x^{2}}=-\sum_{n=1}^{\infty}\left(\frac{n \pi}{L}\right)^{2}\left(A_{n} \cos \alpha_{n} t+B_{n} \sin \alpha_{n} t\right) \sin \frac{n \pi x}{L} .
\end{gathered}
$$

From the shape described by the wavefunction given in Equation (2) we observe that at each moment of time the vibrating string appears as a $1 \mathrm{D}$ differentiable manifold which is a geometrical object embedded in a 2D Euclidean space. In fact, the wavefunction given in Equation (2) can be used to construct the geometric structure of the vibrating string. In differential geometry, the curvature $\kappa$ of a plane curve described by the equation $y=\psi(x)$ is found as [5]

$$
\kappa=\left(1+\left(\frac{d \psi}{d x}\right)^{2}\right)^{-\frac{3}{2}} \frac{d^{2} \psi}{d x^{2}}
$$

Using Equations (5) and (6), the curvature $\kappa$ is rewritten as

$$
\begin{aligned}
\kappa= & \left(1+\left(\sum_{n=1}^{\infty}\left(\frac{n \pi}{L}\right)\left(A_{n} \cos \alpha_{n} t+B_{n} \sin \alpha_{n} t\right) \cos \frac{n \pi x}{L}\right)^{2}\right)^{-\frac{3}{2}} \\
& \times\left(-\sum_{n=1}^{\infty}\left(\frac{n \pi}{L}\right)^{2}\left(A_{n} \cos \alpha_{n} t+B_{n} \sin \alpha_{n} t\right) \sin \frac{n \pi x}{L}\right) .
\end{aligned}
$$

This result shows that the geometric structure of the vibrating string can be described by a classical wavefunction. In other words, wavefunctions can be considered as representations of physical objects. It is also interesting to observe the following. An observer in a space with dimension greater than 1 can see the whole shape of the vibrating string. But for an observer who is a $1 \mathrm{D}$ physical object living on the $x$-axis, then according to this observer the vibrating string appears as an oscillating motion of a single particle, which is of course the appearance on the $x$-axis of all particles that join together to form the string one after another with respect to time. On the other hand, if the vibrating string is in motion in space then it can be seen as a particle. With a suitable experimental setup, this moving vibrating string may be detected as a wave. And furthermore, it can also generate a physical wave if the space is a medium. Although these observations for the vibrating string are obvious but, as will be discussed later, they may not be that obvious when we discuss similar situations for the case when the vibrating object is three-dimensional and the wavefunctions are identified with three-dimensional differentiable manifolds that represent quantum particles. However, to obtain a closer picture to the threedimensional situation, we will give in details in the next section the wave motion of a vibrating circular membrane.

\section{Two-dimensional Wave Associated with a Vibrating Circular Membrane}

In this section we will extend our discussions in Section 2 to the two-dimensional wave that is associated with the vibration of a circular membrane. In general, the wave dynamics of a physical system in two-dimensional space can be described by a wave equation written in the Cartesian coordinates $(x, y)$ as

$$
\frac{1}{c^{2}} \frac{\partial^{2} \psi}{\partial t^{2}}-\frac{\partial^{2} \psi}{\partial x^{2}}-\frac{\partial^{2} \psi}{\partial y^{2}}=0 .
$$

In particular, Equation (9) can be used to describe the dynamics of a vibrating membrane in the $(x, y)$-plane. If the membrane is a circular membrane of radius $a$ then the domain $D$ is given as $D=\left\{x^{2}+z^{2}<a^{2}\right\}$. In the polar coordinates given in terms of the Cartesian coordinates $(x, y)$ as $x=r \cos \theta, y=r \sin \theta$, the two-dimensional wave equation given in Equation (9) is rewritten as

$$
\frac{1}{c^{2}} \frac{\partial^{2} \psi}{\partial t^{2}}-\frac{\partial^{2} \psi}{\partial r^{2}}-\frac{1}{r} \frac{\partial \psi}{\partial r}-\frac{1}{r^{2}} \frac{\partial^{2} \psi}{\partial \theta^{2}}=0 .
$$

The general solution to Equation (10) for the vibrating circular membrane with the condition $\psi=0$ on the boundary of $D$ can be found as [6]

$$
\begin{aligned}
& \psi(r, \theta, t)=\sum_{m=1}^{\infty} J_{0}\left(\sqrt{\lambda_{0 m}} r\right)\left(\begin{array}{l}
C_{0 m} \cos \sqrt{\lambda_{0 m}} c t \\
+D_{0 m} \sin \sqrt{\lambda_{0 m}} c t
\end{array}\right) \\
& \left.+\sum_{m, n=1}^{\infty} J_{n}\left(\sqrt{\lambda_{n m} r}\right)\left(\begin{array}{l}
A_{n m} \cos n \theta \\
+B_{n m} \sin n \theta
\end{array}\right)\left(\begin{array}{l}
C_{n m} \cos \sqrt{\lambda_{n m}} c t \\
+D_{n m} \sin \sqrt{\lambda_{n m}} c t
\end{array}\right)\right)
\end{aligned}
$$

where $J_{n}\left(\sqrt{\lambda_{n m}} r\right)$ is the Bessel function of order $n$ and the quantities $A_{n m}, B_{n m}, C_{n m}$ and $D_{n m}$ can be specified by the initial and boundary conditions. It is also observed that at each moment of time the vibrating membrane appears 
as a 2D differentiable manifold which is a geometric object whose geometric structure can be constructed using the wavefunction given in Equation (11). We now show that the curvature of the surfaces obtained from the vibrating membrane at each moment of time can also be expressed in terms of the derivatives of the wavefunction given in Equation (11). In differential geometry, the Ricci scalar curvature $R$ is shown to be related to the Gaussian curvature $K$ by the relation $R=2 K$, where $K$ is expressed in terms of the principal radii $k_{1}$ and $k_{2}$ of the surface as $K=1 / k_{1} k_{2}$. Consider a surface defined by the relation $z=\psi(x, y)$ in Cartesian coordinates $(x, y, z)$. The Ricci scalar curvature $R$ can be found as [5]

$$
R=\frac{2\left(\psi_{11} \psi_{22}-\left(\psi_{12}\right)^{2}\right.}{\left(1+\psi_{1}^{2}+\psi_{2}^{2}\right)^{2}}
$$

where $\psi_{\mu}=\partial \psi / \partial x^{\mu}$ and $\psi_{\mu \nu}=\partial^{2} \psi / \partial x^{\mu} \partial x^{\nu}$. It is seen that the wavefunction $\psi(r, \theta, t)$ that is obtained from the wave equation given in Equation (11) can be used to determine the Ricci scalar curvature of a surface, which shows that the geometric structure of the vibrating membrane can be described by a classical wavefunction. In other words, as in the case of the vibrating string, wavefunctions that describe the wave motion of a vibrating membrane can be considered as a representation of physical objects. For the benefit of representation in the next section we now give a brief discussion on the geometric formation of quantum particles from a wave equation. We assumed that the circular membrane is made up of particles which are connected with each other by an elastic force. As will be discussed in more details on geometric interactions in section 6 , this assumption leads to a more general hypothesis that a vibrating object is made up of mass points that join together by contact forces. When the membrane vibrates it takes different shapes at each moment of time. Each shape is a 2D differentiable manifold that is embedded in the threedimensional Euclidean space. Now, if we consider the whole vibrating membrane as a particle then its geometric structure is described by the wavefunction $\psi$. It is a timedependent hypersurface embedded in a three-dimensional Euclidean space. Now imagine an observer who is a twodimensional object living in the plane $(x, y)$ and who wants to investigate the geometric structure of the vibrating membrane. Even though he would not be able to observe the shapes of the embedded 2D differentiable manifolds in the three-dimensional Euclidean space, he would still be able to calculate the value of the wavefunction $\psi$ at each point $(x, y)$ that belongs to the domain $x^{2}+z^{2}<a^{2}$. What would the observer think of the nature of the wavefunction $\psi$ ? Does it represent a mathematical object, such as a third dimension, or a physical one, such as fluid pressure? Firstly, because the wavefunction $\psi$ is a solution of a wave equation therefore it must be a wave. Secondly, if the observer who is a $2 \mathrm{D}$ physical object and who does not believe in higher dimensions then he or she would conclude that the wavefunction $\psi$ should only be used to describe events of physical existence other than space and time. In the next section we will show that this situation may in fact be that of the wave-particle duality that we are encountering in quantum physics when our view of the physical existence is restricted to that of a $3 \mathrm{D}$ observer. It is also observed that according to the $2 \mathrm{D}$ observer who is living on the $(x, y)$-plane, the vibrating membrane appears as an oscillating motion of a single string. If the vibrating string is set in motion in space then it can be seen as a particle. With a suitable experimental setup, the moving vibrating membrane may be detected as a wave. And furthermore, it can also generate a physical wave if the space is a medium.

\section{Three-dimensional Wave Associated with a Vibrating Solid Ball}

In this section we want to show that it is possible to identify quantum particles with $3 \mathrm{D}$ differentiable manifolds which in turns are described by the wavefunctions which are solutions of a wave equation. In classical physics, the three-dimensional wave equation written in Cartesian coordinates $(x, y, z)$ of the form

$$
\frac{1}{c^{2}} \frac{\partial^{2} \psi}{\partial t^{2}}-\frac{\partial^{2} \psi}{\partial x^{2}}-\frac{\partial^{2} \psi}{\partial y^{2}}-\frac{\partial^{2} \psi}{\partial z^{2}}=0
$$

can be used to describe the wave motion of different physical fields. However, if we want to generalise the above discussions for $1 \mathrm{D}$ and $2 \mathrm{D}$ wave equations that describe a vibrating string and a vibrating membrane respectively then what geometrical characteristic should we assign to the wavefunction $\psi$ ? Since in $1 \mathrm{D}$ and 2D wave equations, the wavefunction $\psi$ are the actual height of the particles that form the medium which can be viewed in the second and third dimension, respectively, of the space in which they are embedded, therefore we may suggest that the wavefunction $\psi$ in $3 \mathrm{D}$ should also be given the meaning of the height of the particles that form the medium. However, if we want to give the meaning of the height to the 3D wavefunction then the space in which the $3 \mathrm{D}$ vibrating object is embedded must be extended to a four-dimensional Euclidean space. Whether such extension can be justified is a subject that requires further investigation and in fact this can be shown to be related to the fundamental question of why we exist as 3D physical objects. Now, consider a region $D$ which is embedded in a three-dimensional Euclidean space and bounded by a closed surface. As in the case of the string and the membrane considered above, we assume that the region $D$ is a physical object that is made up of mass points joined together by contact forces so that it can vibrate. In general, the region $D$ can be any shape, however, as an illustration let us consider a simple case of which the region $D$ is a solid ball embedded in the $(x, y, z)$-space defined by the relation $D=\left\{x^{2}+y^{2}+z^{2}<a^{2}\right\}$ with the condition $\psi=0$ on the boundary of $D$. In a three-dimensional Euclidean space, such physical objects can only be assumed to vibrate internally inside the solid ball and the mathematical object represented by the function $\psi$ can only be assumed to be a physical entity, such as fluids and acoustics. However, as in the case of the string considered in Section 2 in which the mass points of the string can vibrate into the second dimension of the two-dimensional Euclidean space and that of the membrane considered in 
Section 3 in which the mass points of the membrane can vibrate into the third dimension of the three-dimensional Euclidean space, we may assume that the mass points that form the physical object contained in the region $D=\left\{x^{2}+y^{2}+z^{2}<a^{2}\right\}$ can vibrate into the fourth dimension of a four-dimensional Euclidean space, therefore the mathematical object $\psi$ represents a spatial dimension. When vibrating, at each moment of time, the solid ball becomes a three-dimensional differentiable manifold that is embedded in a four-dimensional Euclidean space. In this case, an observer who is a 3D physical object can only observe the cross-section which is the intersection of the time-dependent differentiable manifold and the three-dimensional Euclidean space into which that the observer is embedded. And the cross-section appears as a $3 \mathrm{D}$ wave to the $3 \mathrm{D}$ observer. Written in the spherical polar coordinates, which are defined in terms of the Cartesian coordinates $(x, y, z)$ as $x=r \sin \theta \cos \phi, \quad y=r \sin \theta \sin \phi, \quad z=r \cos \theta$, the three-dimensional wave equation given in Equation (13) becomes

$$
\begin{aligned}
& \frac{1}{c^{2}} \frac{\partial^{2} \psi}{\partial t^{2}}-\frac{\partial^{2} \psi}{\partial r^{2}}-\frac{2}{r} \frac{\partial \psi}{\partial r} \\
& -\frac{1}{r^{2} \sin \theta} \frac{\partial}{\partial \theta}\left(\sin \theta \frac{\partial \psi}{\partial \theta}\right)-\frac{1}{r^{2} \sin ^{2} \theta} \frac{\partial^{2} \psi}{\partial \phi^{2}}=0 .
\end{aligned}
$$

The general solution to Equation (14) for the vibrating solid ball with a given initial condition can be found by separating the variables in the form $\psi(r, \theta, \phi, t)=$ $S(r, \theta, \phi) T(t)[6]$

$$
\begin{aligned}
& \psi(r, \theta, \phi, t) \\
& =\sum_{l=0}^{\infty} \sum_{j=1}^{\infty} \sum_{m=-l}^{l} A_{l m j} e^{-k \lambda_{l j} t} \frac{J_{l+\frac{1}{2}\left(\sqrt{\lambda_{l j}} r\right)}}{\sqrt{r}} P_{l}^{m}(\cos \theta) e^{i m \phi}
\end{aligned}
$$

where $P_{l}^{m}(\cos \theta)$ is the associated Legendre function and $J_{l+\frac{1}{2}}\left(\sqrt{\lambda_{l j}} r\right)$ is the Bessel function. The wavefunction given in Equation (15) is the general time-dependent shape of the vibrating solid ball embedded in the four-dimensional Euclidean space. Similar to the vibrating string and the vibrating membrane, at each moment of time the vibrating solid ball appears as a 3D differentiable manifold which is a geometric object whose geometric structure can be constructed using the wavefunction given in Equation (15) and can be identified with a quantum particle. Therefore, what we observe as a wave may in fact be a particle and this kind of dual existence may be related to the problem of wave-particle duality we encounter in quantum mechanics. A simpler case is that of a quantum particle that appears as a spherical wave. In this case the wave equation given in Equation (14) reduces to

$$
\frac{1}{c^{2}} \frac{\partial^{2} \psi}{\partial t^{2}}-\frac{\partial^{2} \psi}{\partial r^{2}}-\frac{2}{r} \frac{\partial \psi}{\partial r}=0 .
$$

The general solution to Equation (16) can be found as

$$
\psi(r, t)=\frac{c_{1} f(r-c t)+c_{2} g(r+c t)}{r} .
$$

The above wavefunctions describe the geometric structures of quantum particles as differentiable manifolds embedded in a four-dimensional Euclidean space, therefore, if the Ricci scalar curvature of the vibrating solid ball can be formulated in terms of the wavefunction $\psi$ and its derivatives then the geometric structure of the vibrating solid ball can be determined. In the next section we will show how such relation can be realised for the case of the hydrogen atom when the Ricci scalar curvature can be constructed from the Schrödinger wavefunctions in wave machanics.

\section{Further Discussions on the Geometric Structures of Quantum Particles}

In this section we will outline our previous works on how to invoke Schrödinger wavefunctions in quantum mechanics to construct mathematical structures for quantum particles which are assumed to possess the geometric and topological structures of a three-dimensional differentiable manifold. As shown in our works on spacetime structures of quantum particles [2], the three main dynamical descriptions of physical events in classical physics, namely Newton mechanics, Maxwell electromagnetism and Einstein gravitation, can be formulated in the same general covariant form and they can be represented by the following general equation

$$
\nabla_{\beta} M=k J
$$

where $M$ is a mathematical object that represents the corresponding physical system and $\nabla_{\beta}$ is a covariant derivative. For Newton mechanics, we have $M=E=$ $\frac{1}{2} m \sum_{\mu=1}^{3}\left(d x^{\mu} / d t\right)^{2}+V$ and $J=0$. For Maxwell electromagnetism, $\quad M=F^{\alpha \beta}=\partial^{\mu} A^{v}-\partial^{v} A^{\mu}$ with the four-vector potential $A^{\mu} \equiv(V, \mathbf{A})$ and $J$ can be identified with the electric and magnetic currents. And for Einstein gravitation, $M$ is the Ricci tensor $R^{\alpha \beta}$ and $J$ can be defined in terms of a metric and Ricci scalar curvature. It is shown in differential geometry that the Ricci tensor $R^{\alpha \beta}$ satisfies the Bianchi identities

$$
\nabla_{\beta} R^{\alpha \beta}=\frac{1}{2} g^{\alpha \beta} \nabla_{\beta} R
$$

where $R=g^{\alpha \beta} R_{\alpha \beta}$ is the Ricci scalar curvature [7]. Even though Equation (19) is purely geometrical, it has a covariant form similar to the electromagnetic tensor $\partial_{\alpha} F^{\alpha \beta}=\mu j^{\beta}$ defined in Euclidean space. If the quantity $\frac{1}{2} g^{\alpha \beta} \nabla_{\beta} R$ can be identified as a physical entity, such as a four-current of gravitational matter, then Equation (19) has the status of a dynamical law of a physical theory. In this case a four-current $j^{\alpha}=\left(\rho, \mathbf{j}_{i}\right)$ can be defined purely geometrical as

$$
j^{\alpha}=\frac{1}{2} g^{\alpha \beta} \nabla_{\beta} R
$$

If we use the Bianchi identities as field equations for the gravitational field then Einstein field equations, as in the case of the electromagnetic field, can be regarded 
as a definition for the energy-momentum tensor for the gravitational field, $R_{\mu v}-\frac{1}{2} R g_{\mu v}+\Lambda g_{\mu v}=\kappa T_{\mu v}$. For a purely gravitational field in which $\frac{1}{2} g^{\alpha \beta} \nabla_{\beta} R=0$, the proposed field equations given in Equation (19) also give rise to the same results as those obtained from Einstein formulation of the gravitational field. For a purely gravitational field, Equation (19) reduces to the equation

$$
\nabla_{\beta} R^{\alpha \beta}=0 \text {. }
$$

From Equation (21), we can obtain solutions found from the original Einstein field equations, such as Schwarzschild solution, by observing that since $\nabla_{\mu} g^{\alpha \beta} \equiv$ 0, Equation (21) implies

$$
R_{\alpha \beta}=\Lambda g_{\alpha \beta}
$$

where $\Lambda$ is an undetermined constant. Furthermore, the intrinsic geometric Ricci flow that was introduced by Hamilton can also be derived from Equation (21) and given as follows

$$
\frac{\partial g_{\alpha \beta}}{\partial t}=-2 R_{\alpha \beta}
$$

Mathematically, the Ricci flow is a geometric process that can be employed to smooth out irregularities of a Riemannian manifold [8,9]. From the four-current of matter given in Equation (20), by letting $\alpha=0$, we obtain the matter density component

$$
\rho=j^{0}=\frac{1}{2} g^{0 \beta} \nabla_{\beta} R=\frac{1}{2} g^{0 \beta} \partial_{\beta} R .
$$

In fact, as shown in our works on space time structures of quantum particles, by comparing Equation (24) with the Poisson equation for a potential $V$ in classical physics $\nabla^{2} V=4 \pi \rho$ we can identify the scalar potential $V$ with the Ricci scalar curvature as

$$
V=k R
$$

where $k$ is an undetermined dimensional constant. In the following, using the relation between the potential and the Ricci scalar curvature given in Equation (25), we will show that the Ricci scalar curvature $R$ can be constructed from the wavefunctions obtained from the Schrödinger wave equation in wave mechanics. In his original works, Schrödinger introduced a new function $\psi$, which is real, single-valued and twice differentiable, through the relation $S=\hbar \ln \psi$, where the action $S$ is defined by $S=\int L d t$ and $L$ is the Lagrangian defined by $L=T-\varphi$ with $T$ is the kinetic energy and $\varphi$ is the potential energy. By applying the principle of least action defined in classical dynamics, Schrödinger arrived at the wave equation to describe the stationary state of the hydrogen atom

$$
\nabla^{2} \psi+\frac{2 m}{\hbar^{2}}\left(E+\frac{k q^{2}}{r}\right) \psi=0
$$

Now we show that Schrödinger wavefunction $\psi$ can be used to construct the Ricci scalar curvature associated with the spacetime structures of the quantum states of the hydrogen atom. By using the defined relations $L=d S / d t$, $d S / d t=\partial_{t} S+\sum_{\mu=1}^{3} \partial_{\mu} S\left(\frac{d x^{\mu}}{d t}\right), \quad T=m \sum_{\mu=1}^{3}\left(d x^{\mu} / d t\right)^{2}$ and $\varphi=T-L$, the following relation can be obtained

$$
\varphi=m \sum_{\mu=1}^{3}\left(d x^{\mu} / d t\right)^{2}-\hbar \frac{\partial_{t} \psi+\sum_{\mu=1}^{3} \partial_{\mu} \psi\left(d x^{\mu} / d t\right)}{\psi}
$$

From the relations $V=k \mathrm{R}$ and $V=\varphi / m$, we obtain the following relationship between the Schrödinger wavefunction $\psi$ and the Ricci scalar curvature $\mathrm{R}$

$$
\mathrm{R}=\frac{1}{k}\left(\sum_{\mu=1}^{3}\left(d x^{\mu} / d t\right)^{2}-\frac{\hbar}{m} \frac{\partial_{t} \psi+\sum_{\mu=1}^{3} \partial_{\mu} \psi\left(d x^{\mu} / d t\right)}{\psi}\right)
$$

It is seen that the Schrödinger wavefunction $\psi$ that is obtained from the wave equation given in Equation (26) can be used to determine the Ricci scalar curvature of a three-dimensional differentiable manifold that is identified with the stationary states of a hydrogen atom. In this case of the Schrödinger wavefunction that describes the wave motion of the electron of the hydrogen atom can be considered as a representation of a physical object. In spherical polar coordinates $(r, \theta, \phi)$, the Ricci scalar curvature given in Equation (28) takes the form

$$
\mathrm{R}=\frac{1}{k}\left(\begin{array}{l}
\left(\frac{d r}{d t}\right)^{2}+r^{2} \sin ^{2} \phi\left(\frac{d \theta}{d t}\right)^{2}+r^{2}\left(\frac{d \phi}{d t}\right)^{2} \\
-\frac{\hbar}{m} \frac{\partial_{t} \psi+\partial_{r} \psi \frac{d r}{d t}+\partial_{\theta} \psi \frac{d \theta}{d t}+\partial_{\phi} \psi \frac{d \phi}{d t}}{\psi}
\end{array}\right)
$$

The eigen functions $\psi_{n l m}(r, \theta, \phi)$ representing the stationary states of the hydrogen atom, which are solutions to the Schrödinger wave equation given in Equation (26), can be found in the form $\psi_{n l m}(r, \theta, \phi)=R_{n l}(r) Y_{l m}(\theta, \phi)$, where the spherical harmonics $Y_{l m}(\theta, \phi)$ and the radial functions $R_{n l}(r)$ are given as

$$
\begin{gathered}
Y_{l m}(\theta, \phi)=(-1)^{m}\left(\frac{(2 l+1)(l-m) !}{4 \pi(l+m) !}\right)^{\frac{1}{2}} P_{l}^{m}(\cos \theta) e^{i m \phi}(30) \\
R_{n l}(r)=-\left(\left(\frac{2}{n a_{0}}\right)^{3} \frac{(n-l-1) !}{2 n((n+l) !)^{3}}\right)^{\frac{1}{2}} e^{-\frac{\rho}{2}} \rho^{l} L_{n+l}^{2 l+1}(\rho)(31)
\end{gathered}
$$

where $\rho=2 r / n a_{0}$ and $a_{0}=4 \pi \varepsilon_{0} \hbar^{2} / m q^{2}$ [10]. From these solutions, the first few normalised wavefunctions for the stationary states of the hydrogen atom and their corresponding Ricci scalar curvatures are given below

$$
\begin{gathered}
\psi_{100}(r, \theta, \phi)=\frac{1}{\sqrt{\pi}}\left(\frac{1}{a_{0}}\right)^{\frac{3}{2}} e^{-\frac{r}{a_{0}}} \\
\mathrm{R}=\frac{1}{k_{3}}\left(\begin{array}{l}
\left(\frac{d r}{d t}\right)^{2}+r^{2} \sin ^{2} \phi\left(\frac{d \theta}{d t}\right)^{2} \\
+r^{2}\left(\frac{d \phi}{d t}\right)^{2}+\frac{\hbar}{m a_{0}} \frac{d r}{d t}
\end{array}\right)
\end{gathered}
$$




$$
\begin{gathered}
\psi_{200}(r, \theta, \phi)=\frac{1}{4 \sqrt{2 \pi}}\left(\frac{1}{a_{0}}\right)^{\frac{3}{2}}\left(2-\frac{r}{a_{0}}\right) e^{-\frac{r}{2 a_{0}}} \\
\mathrm{R}=\frac{1}{k_{3}}\left(\begin{array}{l}
\left.\left(\frac{d r}{d t}\right)^{2}+r^{2} \sin ^{2} \phi\left(\frac{d \theta}{d t}\right)^{2}\right) \\
+r^{2}\left(\frac{d \phi}{d t}\right)^{2}+\frac{3 \hbar}{m a_{0}} \frac{d r}{d t}
\end{array}\right) \\
\psi_{300}(r, \theta, \phi)=\frac{1}{81 \sqrt{3 \pi}}\left(\frac{1}{a_{0}}\right)^{\frac{3}{2}}\left(27-\frac{18 r}{a_{0}}+\frac{2 r^{2}}{a_{0}^{2}}\right) e^{-\frac{r}{3 a_{0}}} \\
\mathrm{R}=\frac{1}{k_{3}}\left(\begin{array}{l}
\left(\frac{d r}{d t}\right)^{2}+r^{2} \sin ^{2} \phi\left(\frac{d \theta}{d t}\right)^{2} \\
\left.+r^{2}\left(\frac{d \phi}{d t}\right)^{2}+\frac{\hbar}{m}\left(\frac{54-4 r}{a_{0}}\right) \frac{d r}{d t}\right)
\end{array}\right.
\end{gathered}
$$

As a further remark, we want to mention here that besides the covariant form given in Equation (18), Maxwell field equations of electromagnetism can also be shown to have the same quantum formulation as Dirac relativistic equation of quantum mechanics. We have shown that both Maxwell field equations and Dirac equation can be formulated covariantly from a general system of linear first order partial differential equations [11,12,13]. A matrix form of a system of linear first order partial differential equations can be written as follows [14]

$$
\left(\sum_{i=1}^{n} A_{i} \frac{\partial}{\partial x_{i}}\right) \psi=k_{1} \sigma \psi+k_{2} J
$$

where $\psi=\left(\psi_{1}, \psi_{2}, \ldots, \psi_{n}\right)^{T}, A_{i}, \sigma$ and $J$ are matrices, and $k_{1}$ and $k_{2}$ are undetermined constants. Now, if we apply the operator $\sum_{i=1}^{n} A_{i} \frac{\partial}{\partial x_{i}}$ on the left on both sides of Equation (38) and if we assume further that the matrices $A_{i}$ and $\sigma$ are constant such that $A_{i} \sigma=\sigma A_{i}$, then Equation (38) becomes

$$
\begin{aligned}
& \left(\sum_{i=1}^{n} A_{i}^{2} \frac{\partial^{2}}{\partial x_{i}^{2}}+\sum_{i=1}^{n} \sum_{j>i}^{n}\left(A_{i} A_{j}+A_{j} A_{i}\right) \frac{\partial^{2}}{\partial x_{i} \partial x_{j}}\right) \psi \\
& =k_{1}^{2} \sigma^{2} \psi+k_{1} k_{2} \sigma J+k_{2} \sum_{i=1}^{n} A_{i} \frac{\partial J}{\partial x_{i}} .
\end{aligned}
$$

In order for the above systems of partial differential equations to be used to describe physical phenomena, the matrices $A_{i}$ must be determined. We have shown that for both Dirac and Maxwell field equations, the matrices $A_{i}$ must take a form so that Equation (39) reduces to the following equation

$$
\left(\sum_{i=1}^{n} A_{i}^{2} \frac{\partial^{2}}{\partial x_{i}^{2}}\right) \psi=k_{1}^{2} \sigma^{2} \psi+k_{1} k_{2} \sigma J+k_{2} \sum_{i=1}^{n} A_{i} \frac{\partial J}{\partial x_{i}} .
$$

Our method of derivation of Dirac equation from a system of linear first order particle differential equations may be seen as a classical formulation and this is in contrast to different methods of derivation of Dirac equation within the current framework of quantum mechanics. For example, it has been shown that Dirac equation can be derived from the main equation of relativistic canonical quantum mechanics [15]. From the above discussions it seems that physics can be formulated purely in terms of differential geometry and topology, and to strengthen this view of physical formulation we will discuss in the next section whether physical interactions may in fact simply be geometrical processes.

\section{A Discussion on Geometric Interactions}

As being assumed in the above sections that vibrating physical objects are formed from mass points by contact forces, with regard to this view of physical existence in this section we will discuss further how mass points and contact forces can be assumed and formulated in terms of geometric interactions according to decomposed $n$-cells from a CW complex when we consider quantum particles as differentiable manifolds and physical interactions are identified with geometrical processes [16]. In general, we may consider quantum particles as differentiable manifolds of dimension $n$ which can emit submanifolds of dimension $m \leq n$ by decomposition. However, in order to formulate a physical theory we would need to devise a mathematical framework that allows us to account for the amount of subspaces that are emitted or absorbed by an elementary particle, which are assumed to be a differentiable manifold. This assumption leads to the visualisation of quantum particles as $\mathrm{CW}$ complexes which are constructed from $m$-dimensional closed cells topologically glued together through the operation of connected sum. The effect of the operation results in a joint of two given manifolds. More generally, manifolds can be glued together along submanifolds. Let $M_{1}$ and $M_{2}$ be two smooth oriented manifolds of equal dimension and $V$ a smooth closed oriented manifold embedded as a submanifold into both $M_{1}$ and $M_{2}$. The connected sum of $M_{1}$ and $M_{2}$ along $V$ is then the space $\left(M_{1}, V\right) \#\left(M_{2}, V\right)$ [17]. For the case of three-dimensional manifolds, the decomposition will produce three types of prime manifolds, which are the spherical types, $S^{2} \times S^{1}$ and $K(\pi, 1)$. Only the prime manifold $K(\pi, 1)$ can be decomposed along embedded tori [18]. In order to describe the evolution of a geometric process as a physical interaction we assume that an assembly of cells of a specified dimension will give rise to a certain form of physical interactions and the intermediate particles, which are the force carriers of physical fields decomposed during a geometric evolution, may possess the geometric structures of the $n$-spheres and the $n$-tori. This speculation leads to a more profound speculation that physical properties assigned to an elementary particle, such as charge, are in fact manifestations due to the force carriers rather than physical quantities that are contained inside the elementary particle. If this is the case then the analysis of physical interactions will be reduced to the analysis of the geometrical processes that are related to the geometric structures of the force carriers. Therefore, for observable physical phenomena, the study of physical dynamics reduces to the study of the geometric evolution of differentiable manifolds. In particular, if an elementary 
particle is considered to be a three-dimensional manifold then there are four different types of physical interactions that are resulted from the decomposition of 0 -cells, 1-cells, 2-cells and 3-cells, and we will discuss this situation further in the following.

Forces associated with 0-cells: For a definite perception of a physical existence, we assume that space is occupied by mass points which interact with each other through the decomposition of 0 -cells. However, since 0 cells have dimension zero therefore there is only contact forces between the mass points, which may be assumed to be constant for a short range, $F=k_{0}$. When the mass points join together through the contact forces they form elementary particles. The 0 -cells with contact forces can be arranged to form a particular topological structure [19].

Forces associated with 1-cells: Depending on the topological structure of the cells it is possible to devise different forms of force associated with the cells. For the case of 1-cells, it is anticipated that they will manifest either as a linear force $F \sim r$ or a force of inverse law $F \sim 1 / r$ or a combination of the two

$$
\begin{gathered}
\mathbf{F}=k_{1} \mathbf{r} \\
\mathbf{F}=\frac{k_{2} \mathbf{r}}{r^{2}} \\
\mathbf{F}=\left(k_{1}+\frac{k_{2}}{r^{2}}\right) \mathbf{r} .
\end{gathered}
$$

Forces associated with 2-cells: The decomposed 2-cells from an elementary particle can manifest either as a square force $F \sim r^{2}$ or a force of inverse square law $F \sim 1 / r^{2}$ or a combination of the two

$$
\begin{gathered}
\mathbf{F}=k_{3} r \mathbf{r} \\
\mathbf{F}=\frac{k_{4} \mathbf{r}}{r^{3}} \\
\mathbf{F}=\left(k_{3} r+\frac{k_{4}}{r^{3}}\right) \mathbf{r}
\end{gathered}
$$

Forces associated with 3-cells: For the decomposition of 3-cells from a manifold, even though it should be considered as a manifestation of either a cube force $F \sim r^{3}$ or a force of inverse cube law $F \sim 1 / r^{3}$ or a combination of the two, however, this form of geometric interaction can be applied to explain the cosmological evolution in general relativity. The cube force and the inverse cube law are given as

$$
\begin{gathered}
\mathbf{F}=k_{5} r^{2} \mathbf{r} \\
\mathbf{F}=\frac{k_{6} \mathbf{r}}{r^{4}} \\
\mathbf{F}=\left(k_{5} r^{2}+\frac{k_{6}}{r^{4}}\right) \mathbf{r} .
\end{gathered}
$$

From the above considerations, we can assume a general force which is a combination of those forces resulted from the decomposition of $n$-cells of all dimensions. For the case of dimension three, the general force takes the form

$$
F=\sum_{n=-3}^{3} k_{n} r^{n}
$$

where $k_{n}$ are constants which can be determined from physical considerations.

For the case of physical interactions that are associated with the decomposition of 3-cells from a differentiable manifold, the physical interactions that are associated with the evolution of the geometric processes can be formulated in terms of general relativity. Physically, we can visualise with a complete picture how 1-cells and 2-cells are formed and released from a 3-dimensional manifold, but for the case of forming and releasing a 3-cell from a 3-dimensional manifold $M$, such complete visualisation seems to be beyond our physical ability, except for local observation. This is similar to the visualisation of three-dimensional wavefunctions that represent three-dimensional differentiable manifolds as discussed in Section 4. Mathematically, the forming and releasing of a 3-cell from a 3-dimensional manifold $M$ can be expressed as a decomposition in the form $M=M \# S^{3}$. We assume that the physical interactions associated with the forming and releasing of 3-cells are geometric processes that smooth out irregularities of the intrinsic geometric structure of the manifold. The geometric irregularities can be viewed physically as an inhomogeneous distribution of matter in space and the forming and releasing of the $S^{3}$ cells as an expansion. A similar geometric process that smooths out an inhomogeneous distribution of a substance can be realised on the surface of a 2-dimensional sphere. In order to smooth out the irregularities, 1-cells in the form of circles can be formed and released from a position with dense substance and the geometric process is viewed as a local expansion. With this realisation, the geometric process of decomposition of 3-cells $S^{3}$ to smooth out irregularities of the distribution of matter in the observable universe can be formulated in terms of general relativity in which the change of intrinsic geometric structures of the manifold is due to the change of mathematical objects that define the manifold. These mathematical objects are perceived as physical entities like the energy-momentum tensor and the equations that describe the changes can be obtained from mathematical identities, such as Bianchi identities, the Ricci flow, or Einstein field equations of general relativity given as

$$
R_{\mu v}-\frac{1}{2} R g_{\mu v}+\Lambda g_{\mu v}=\kappa T_{\mu v}
$$

with the pseudo-Euclidean Robertson-Walker metric [20]

$$
d s^{2}=c^{2} d t^{2}-S^{2}(t)\left(\frac{d r^{2}}{1-k r^{2}}+r^{2}\left(d \theta^{2}+\sin ^{2} \theta d \phi^{2}\right)\right)(52)
$$

or with the Euclidean Robertson-Walker metric [21]

$$
d s^{2}=c^{2} d t^{2}+S^{2}(t)\left(\frac{d r^{2}}{1-k r^{2}}+r^{2}\left(d \theta^{2}+\sin ^{2} \theta d \phi^{2}\right)\right)
$$

where $k=-1,0,1$. In fact, the geometric interactions can also be extended to temporal manifold in which time has three dimensions and space has one dimension [22], and more general to the case when the spatiotemporal 
manifold can be described as a six-dimensional spherical bundle [23].

\section{Conclusion}

In this work we have discussed the possibility to explain the dual character of a quantum particle in which a quantum object can exhibit both seemingly irreconcilable characters of a physical object defined in classical physics, i.e., the distinguished character that is associated with a particle and that with a wave. Following Einstein's perception that physics should be formulated according to the intrinsic geometric structures of spacetime, similar to the geometric formulation of general relativity, first we assume that quantum particles should possess the geometric and topological structures of 3D differentiable manifolds. Then we show that in order for 3D differentiable manifolds to exhibit wave character they must be solutions of a wave equation so that if quantum particles are identified with these differentiable manifolds then they can manifest wave characteristics. As illustrations, we have examined wave equations that are used to describe the wave motion of physical objects, such as the wave dynamics of a vibrating string and a vibrating membrane, and in particular the vibration of a solid ball. In fact, the main subject that we want to discuss in this work is the wave characteristic of a solid ball by suggesting that the wave motion of a vibrating solid ball described by a wave equation whose solutions are not considered to describe physical phenomena as observed by a 3D observer but differentiable manifolds embedded in a four-dimensional Euclidean space. When the solid ball vibrates into the fourth dimension of a four-dimensional Euclidean space, then the wavefunction can represent a spatial dimension and the solid ball becomes a 3D differentiable manifold embedded in a four-dimensional Euclidean space. In this case, an observer which is a 3D physical object can only observe the cross-section which is the intersection of the time-dependent differentiable manifold and the threedimensional Euclidean space into which that the observer is embedded. And the cross-section appears as a 3D wave to the 3D observer. For a complete description in terms of geometric formulation we also discuss geometric interactions in which decomposed cells from a CW complex could be associated with forces encountered in physical dynamics. In particular we show that all quantum particles can be formed from mass points joined by contact forces which are associated with the decomposition of 0 -cells from a CW complex which is identified with a quantum particle.

\section{Acknowledgements}

I would like to thank the reviewers for their comments and suggestions, and the administration of SciEP for their advices.

\section{References}

[1] Erwin Schrödinger, Collected Papers on Wave Mechanics (AMS Chelsea Publishing, New York, 1982).

[2] A. Einstein, The Principle of Relativity (Dover Publications, New York, 1952).

[3] Vu B Ho, Spacetime Structures of Quantum Particles (Preprint, ResearchGate, 2017), viXra 1708.0192v2, Int. J. Phys. vol 6, no 4 (2018): 105-115.

[4] R. Haberman, Elementary Applied Partial Differential Equations, Prentice-Hall Inc, Sydney, 1987.

[5] E. Kreyszig, Introduction to Differential Geometry and Riemannian Geometry (University of Toronto Press, 1975)

[6] Walter A. Strauss, Partial Differential Equation (John Wiley \& Sons, Inc., New York, 1992).

[7] Ray D’Inverno, Introducing Einstein's Relativity (Clarendon Press, Oxford, 1992).

[8] Richard S. Hamilton, Three-Manifolds with Positive Ricci Curvature, J. Diff. Geo., 17 (1982) 255-306.

[9] Huai-Dong Cao and Xi-Ping Zhu, A Complete Proof of the Poincaré and Geometrization Conjectures-Application of the Hamilton-Perelman Theory of the Ricci Flow, Asian J. Math., Vol 10, No. 2, 165-492, June 2006.

[10] B. H. Bransden and C. J. Joachain, Introduction to Quantum Mechanics (Longman Scientific \& Technical, New York, 1989).

[11] Vu B Ho, Formulation of Maxwell Field Equations from a General System of Linear First Order Partial Differential Equations (Preprint, ResearchGate, 2018), viXra 1802.0055v1.

[12] Vu B Ho, Formulation of Dirac Equation for an Arbitrary Field from a System of Linear First Order Partial Differential Equations (Preprint, ResearchGate, 2018), viXra 1803.0645v1.

[13] Vu B Ho, On Dirac Negative Mass and Magnetic Monopole (Preprint, ResearchGate, 2018), viXra 1806.0319v1.

[14] S. V. Melshko, Methods for Constructing Exact Solutions of Partial Differential Equations, Springer Science \& Business Media, Inc, 2005.

[15] V. M. Simulik and I. Yu. Krivsky, Once more on the derivation of the Dirac equation, arXiv: 1309.0573v2 [math-ph] 22 Sep 2013.

[16] Vu B Ho, A Classification of Geometric Interactions (Preprint, ResearchGate, 2018), viXra 1805.0329v1.

[17] Allen Hatcher, Algebraic Topology, 2001.

[18] K. Yasuno, T. Koike and M. Siino, Thurston's Geometrization Conjecture and cosmological models, arXiv:gr-qc/0010002v1, 2000.

[19] Allen Hatcher and William Thurston, Moduli Spaces of Circle Packings, 2015

[20] Lewis Ryder, Introduction to General Relativity (Cambridge University Press, Melbourne, 2009).

[21] Vu B Ho, Euclidean Relativity (Preprint, ResearchGate, 2017), viXra $1710.0302 v 1$

[22] Vu B Ho, Temporal Geometric Interactions (Preprint, Research Gate, 2018), viXra 1807.0134v1

[23] $\mathrm{Vu} \mathrm{B}$ Ho, On the Geometric Structure of the Spatiotemporal Manifold (Preprint, ResearchGate, 2018), viXra 1808.0144v1. 